\section{RENOVACIÓN, INNOVACIÓN Y TIC EN EL EEES}

\section{Estrella Martínez Rodrigo}

\section{Profesora de Comunicación Audiovisual}

Facultad de Comunicación y Documentación. Universidad de Granada. Campus Universitario de Cartuja, s/n. Granada (España) 18071 Email: emrodrigo@ugr.es

\section{Ana Mª González Fernández}

\section{Investigadora en Comunicación Audiovisual}

Facultad de Comunicación y Documentación. Universidad de Granada. Campus Universitario de Cartuja, s/n. Granada (España) 18071. Email: dima@correo.ugr.es

\section{Resumen}

El Espacio Europeo de Educación Superior, al incluir los créditos ETC (Sistema Europeo de Transferencia de Créditos), cambia la estructura de los planes de estudios y la carga de horas lectivas de los alumnos. En esta nueva situación, se pretende que cualquier estudiante adquiera las competencias necesarias para el ejercicio de su futura profesión. Con este fin, la metodología didáctica que propugna el EEES concede más protagonismo al alumnado, que trabajará de una forma más autónoma, sin depender tanto de las clásicas "clases magistrales" y de los conocimientos adquiridos dentro del espacio físico del aula. Los docentes, por su parte, se convierten en tutores o guías del proceso de aprendizaje de sus pupilos, para lo cual unos y otros disponen de herramientas útiles basadas en las Tecnologías de la Comunicación y

\section{Palabras clave}

Estudios audiovisuales, Espacio Europeo de Educación Superior (EEES), enseñanza, aprendizaje a lo

largo de la vida, TIC

Key Words

Audiovisual Studies, European Higher Education Area (EHEA), teaching,

lifelong learning, ICT

\footnotetext{
Abstract

European Higher Education Area (EHEA), according to the European Credit Transfer System (ECTS), changes both the structure of the University studies and the students' workload, as well. Within, it is intended that students acquire the competences needed in the labour market. Therefore, education and training is focused on pupils who will carry out autonomous work, instead of depending on teachers' 'master class". On the other hand, teachers become mentors who guide their pupils' learning process; for both, teachers and pupils, are available useful Information and Communication Technology (ITC) tools. All the changes introduced by EHEA have advantages and, of course, disadvantages which are analysed in this paper, paying special attention to the activities already applied favourably to the Audiovisual Studies area.
} 
de la Información (TIC). Todos estos cambios propuestos por el EEES presentan múltiples ventajas (y cómo, no, también inconvenientes) que, serán desglosados aquí, prestando especial atención a las iniciativas ya llevadas a cabo, y a los estudios del área Audiovisual.

\section{Introducción}

El 19 de junio de 1999, los Ministros Europeos de Educación subscribieron la declaración de Bolonia, cuyo principal objetivo radica en "incrementar la competitividad del sistema Europeo de educación superior”. Posteriormente, en 2001, ésta se ratificó y amplió con la declaración de Praga, la cual hace hincapié en promover la movilidad de toda la comunidad universitaria para enriquecer los valores "democráticos, diversidad de culturas y lenguas, y la diversidad de los sistemas de educación superior".

Han transcurrido diez años desde que se dio el primer paso hacia la implantación de los nuevos títulos de grado que cambiarán sustancialmente la estructura, organización y metodología de los estudios universitarios. Estamos, pues, en la antesala del Espacio Europeo de Educación Superior (EEES)

La mayoría de los cambios que el EEES plantea se encuadran dentro de una sociedad que también está en continuo proceso de evolución, donde las Tecnologías de la Información y Comunicación (TIC) son cruciales, donde la totalidad de estudiantes universitarios debe adecuarse a las necesidades profesionales de su nuevo entorno, conocido como "la sociedad de la informa- ción” (Machlup, 1962) o como "sociedad red" (Castells, 2007).

Por este motivo, la Universidad debe adoptar y adaptar un método de enseñanza válido para el mundo actual y para un alumnado joven, familiarizado con estas herramientas; de ahí que las TIC sean una de las bazas fundamentales de los próximos planes de estudio, aunque, como veremos a continuación, no serán las únicas.

La configuración del nuevo título en Comunicación Audiovisual también se verá sometido a una serie de actividades formativas adecuadas al estándar europeo que pretende garantizar la homogeneización de los planes de estudio y de las competencias profesionales en los países adheridos a la declaración de Bolonia.

Al margen de las protestas estudiantiles, las polémicas y el debate suscitado por ciertas críticas razonables a esta "convergencia europea", pensamos que el EEES puede mejorar sustancialmente la calidad de la formación universitaria en general y de los estudios de Comunicación Audiovisual en particular, aunque hay que dejar muy claro que ya están en marcha algunos métodos de trabajo en el aula y que no son una novedad exclusiva del Plan Bolonia, sino una reali- 
dad integrada en la metodología docente actual.

Precisamente el propósito de este trabajo es desglosar y describir las medidas que repercuten de manera más positiva para la enseñanza y aprendizaje de los estudios de este ámbito, prestando especial atención al uso de las Nuevas Tecnologías (NNTT) e Internet.

\section{Objetivos}

El primer objetivo es hacer una breve descripción de las nuevas estrategias metodológicas de enseñanza y aprendizaje que el EEES introduce, así como el papel de las TIC y su aplicación en la educación universitaria.

El segundo es analizar la aplicación de algunas de las medidas anteriores en los planes de estudio en Comunicación Audiovisual.
En tercer lugar, comentar algunas experiencias innovadoras que ya están en marcha, puestas al servicio del profesorado y alumnado.

Finalmente, realizar una reflexión constructiva acerca de las desventajas o dificultades de todo lo expuesto, ya que es necesario ser conscientes de los escollos que existen para poder salvarlos.

\section{Metodología}

La metodología empleada para conseguir los objetivos trazados es descriptiva y analítica, siguiendo un punto de vista crítico con respecto a los presupuestos y experiencias didácticas que desarrollan las reformas introducidas por el espacio de convergencia europeo.

Para ello, en primer lugar, se desglosan y explican los conceptos relativos al EEES que suponen una renovación docente considerable. Este punto del artículo está basado en la documentación oficial publicada por los principales agentes implicados en el
EEES, las propias universidades Europeas (en concreto 100), unidas en el proyecto Tuning para elaborar un marco de trabajo y de referencia común, comparable y compatible, donde poder aplicar al curriculum los objetivos del Plan Bolonia.

Por otro lado, se hace un repaso de trabajos e investigaciones anteriores en materia de NNTT y alfabetización para adquirir las habilidades de gestión de la información implicadas en el cambio metodológico que el EEES propone, prestando especial interés a aquellas que ya se han materializado 
en recursos eficientes y útiles para alumnos y profesores aplicables al ámbito de la
Comunicación Audiovisual.

\section{La enseñanza y el aprendizaje en el EEES: conceptos fundamentales y papel de las TIC}

Dentro de este marco tan amplio que constituye la convergencia europea de los estudios superiores, se han desarrollado cambios estructurales que modifican sustancialmente la ordenación académica de los títulos universitarios. Sin embargo, dichos cambios también tienen una repercusión directa en la concepción tradicional de docencia y estudio al que profesorado y alumnado están acostumbrados.

Una de estas modificaciones son los créditos ECTS (el Sistema Europeo de Transferencia de Créditos o según sus siglas en inglés European Credit Transfer System), donde se especifica la carga de trabajo, es decir, las horas que el/la estudiante deben dedicar a las tareas marcadas para conseguir las metas académicas propuestas. La definición de crédito europeo está recogida en el Real Decreto 1125/2003, de 5 de septiembre, por el que se establece el sistema europeo de créditos y el sistema de calificaciones en las titulaciones universitarias de carácter oficial y validez en todo el territorio nacional, que especifica lo siguiente: "El crédito europeo es la unidad de medida del haber académico que representa la cantidad de trabajo del estudiante para cumplir los objetivos del programa de estudios y prácticas $[\ldots] ”$.

El crédito europeo es el principal instrumento empleado por el proyecto Tuning para comparar y promover la compatibilidad de la educación superior en Europa. Su valor constituye el volumen de trabajo del estudiante: su asistencia a clases magistrales, seminarios, tutorías, las tareas que debe hacer en el centro o en su propia casa, prácticas, preparación de exámenes, etc. para aprobar o superar los objetivos propuestos. Se ha establecido que 60 créditos son la carga o volumen de trabajo de un año académico, con lo cual, un crédito europeo está calculado para una carga de trabajo de aproximadamente 25 o 30 horas (lectivas y no lectivas)

A pesar de que este concepto de crédito ECTS pueda parecer un término abstracto y difícil de concretar, en el marco creado por Tuning, se especifica que los objetivos del programa de estudios deben ser elaborados con respecto a las competencias que serán adquiridas y a los resultados del aprendizaje. Por un lado, las competencias se refieren a las capacidades, los conoci- 
mientos o habilidades de diferente índole aplicables al ejercicio de la profesión, mientras que por otro, los resultados del aprendizaje son "manifestaciones de lo que se espera que un estudiante sepa, entienda y sea capaz de demostrar una vez concluido el aprendizaje" (Una introducción a Tuning Educational Structures in Europe, 2006)

Según ambas nociones, en las competencias y resultados del aprendizaje, se aprecian dos particularidades esenciales de la reforma del EEES, casi inseparables entre sí: el carácter eminentemente práctico de los estudios y el vuelco hacia el ámbito empresarial.

El enfoque desde un punto de vista práctico, encaminado a la inserción laboral del alumnado, requiere una reformulación del paradigma tradicional enseñanzaaprendizaje; si, en opinión de Angulo Rasco (2008: 181), en el modelo tradicional había "una educación centrada en la enseñanza, a la que equiparan con la adquisición de contenidos académicos [...]" en la actualidad se quiere alcanzar "una nueva educación superior centrada en el aprendizaje" (en Educar por competencias ¿qué hay de nuevo?) Así, Cabero Almenara indica que la metodología didáctica se ha de basar en:

[...] aprender a aprender lo que sea necesario para el desempeño profesional, las asignaturas se diseñarán de modo que se sitúen en el contexto del título en el que se imparten y se hará un mejor y mayor uso de las tecnologías de la comunicación para trabajar dichas competencias tanto presencial como no presencialmente (Cabero Almenara, 2007: 20)

El papel del profesor será el de un guía o tutor para el alumnado, el cual deberá “aprender a aprender": saber dónde encontrar el conocimiento, ser capaz de estar en permanente proceso de actualización, manejar las fuentes de información de su área específica, comprender y relacionar datos, etc. En definitiva, adaptarse al llamado “aprendizaje a lo largo de la vida” (González y Wagenaar, 2003:126)

Dado que la carga lectiva se reduce en pro del trabajo autónomo por parte del estudiante (lo cual implica el manejar con soltura los medios y entornos digitales de almacenamiento de información) las TIC serán de gran utilidad para el profesorado y alumnado por la amplia gama de materiales y recursos puestos a disposición de unos y otros: plataformas virtuales de gestión y creación de cursos en Internet que permitan la incorporación de un portafolio del estudiante, repositorios de material audiovisual (clips de vídeo con clases magistrales o cualquier otro elemento didáctico) y un largo etcétera donde, sin duda, la palabra clave es la interactividad.

Llegados a este punto, habrá opiniones contrarias que puedan argüir que la interactividad también se producía en las clases magistrales, entre docente y estudiante o que el proceso de consulta de información en la biblioteca constituye un método igualmente válido de aprendizaje continuo; sin embargo, es necesario hacer énfasis que 
el modelo pedagógico perseguido es rotar desde "un modelo de enseñanza despersonalizado y masivo, a uno centrado en su propio proceso de aprendizaje” (González Boticario y Gaudioso Vázquez, 2003) y que el uso de las nuevas tecnologías no persigue desplazar o anular otras formas válidas de aprendizaje a lo largo de la vida, sino complementarlas y adaptarlas a los nuevos retos de la sociedad del conocimiento y, sobre todo, proporcionar a los futuros profesionales, que son los actuales alumnos y alumnas, las herramientas que utilizarán en el ejercicio de su profesión. Motivo por el cual el EEES puede beneficiar concretamente a los estudios de Comunicación Audiovisual.

\section{El Grado de Comunicación Audiovisual: renovación, innovación y TIC}

Los estudios de Comunicación Audiovisual cuentan en Europa y, especialmente en España, con una trayectoria reciente, tal y como constata la Agencia Nacional de la Calidad y Acreditación (Aneca) en el Libro Blanco de los Títulos de Grado en Comunicación (2005). Ello se puede deber a que el sector audiovisual es también un campo relativamente novedoso cuya evolución constante se encuentra determinada por los avances tecnológicos. Es aquí donde la actualización y las NNTT tienen una relevancia crucial: "la evolución de la industria audiovisual, que supone la incorporación de nuevas tecnologías y la progresiva disolución de las fronteras que separaban medios audiovisuales e informática precisan una nueva discusión para intentar actualizar los conocimientos disponibles” (Pestano Rodríguez, 1999).

La necesidad de dicha actualización debe trasladarse al alumnado a lo largo del pro- ceso de aprendizaje para su preparación profesional, pero, es innegable que el profesorado igualmente ha de detectar los cambios o transiciones del sector audiovisual y ser capaces de flexibilizar los contenidos de sus asignaturas para no quedar estancados al margen de la realidad profesional de su ámbito.

Una de las grandes ventajas del Libro Blanco de los Títulos de Grado en Comunicación, es que, para cumplir con su objetivo principal (examinar las tres ramas de los estudios de comunicación actuales y elaborar una "propuesta" que posibilite la adecuación al EEES), se consultaron a los colectivos implicados en el cambio: profesores, estudiantes y "gestores universitarios" (2005: 28), así como a los propios profesionales que ejercen en el sector. De este estudio, resultaron cuatro perfiles profesionales básicos, distribuidos según la temática o especialización de cada uno: 
- director, guionista y realizador audiovisual,

- productor y gestor audiovisual,

- diseño de producción y posproducción visual y sonora,

- investigador, docente y experto en estudios visuales,

para los cuales se detalla una lista muy exhaustiva de conocimientos y competencias concretas que los capacita en el ejercicio de su profesión, clasificados cada uno en "conocimientos disciplinares", "competencias profesionales”, “competencias académicas” y “otras competencias” (2005: 228).

Esta exhaustividad, empero, repercute en la ingente cantidad de conocimientos, habilidades, capacidades y actitudes que los estudiantes en Comunicación Audiovisual deben adquirir en los cuatro años que dura el futuro grado. Ahora bien, lograr lo anterior requiere, por parte del docente, una muy buena planificación, selección de los contenidos y actividades, y una gestión del tiempo adecuada; en otras palabras, profesorado y estudiantes deben hacer "un mejor y mayor uso de las tecnologías de la comunicación para trabajar dichas competencias tanto presencial como no presencialmente" (Cabero Almenara, 2007: 20)

Asimismo, es imprescindible que la información y comunicación sea accesible $\mathrm{y}$ fluida, entre alumnado y profesorado o entre los mismos docentes de diferentes áreas, evitando al máximo la atomización o las divisiones estancas entre miembros de distintos departamentos pero involucrados en el proceso de enseñanza. De nuevo tenemos que referirnos a las NNTT y a las TIC como herramientas fundamentales que ofrecen soluciones muy válidas a estos problemas; soluciones que no son utópicas ni difíciles de conseguir, tal y como veremos a continuación.

\subsection{Algunas herramientas en Internet para docentes $y$ alumnado}

En la actualidad existen a disposición de los docentes un variado número de plataformas webs donde se puede acceder a la información, metodología, actividades, evaluación, etc. de las asignaturas, facilitando el e-learning, teleformación o "aprendizaje a distancia”. Una de las más completas y conocidas es la plataforma Moodle, (cuyo software es libre) basada en las múltiples posibilidades y ventajas que ofrecen otros recursos propios de Internet como los blogs, chats, foros o wikis. Moodle reúne todas las características de la enseñanza a distancia señaladas por Aretio (2001): "separación entre profesor y alumno, apoyo tutorial, aprendizaje independiente y flexible y la comunicación bidireccional" (en González Boticario y Gaudioso Vázquez, 2003: 12). Al mismo tiempo, facilita el trabajo en grupo y el aprendizaje colaborativo entre compañeros y compañeras. 
Molist, en su artículo del periódico El País (2008), describe a Moodle como:

un campus virtual donde el profesor puede distribuir materiales y encuestas a los alumnos; crear foros de debate, glosarios, estadisticas y calendarios de asignaturas; comunicarse con los estudiantes por correo o mensajería instantánea; hacer tutorías electrónicas en privado o en grupo; recoger trabajos; repartir notas; responder dudas de los alumnos; evaluar su participación...

$\mathrm{Su}$ versatilidad ha conseguido que las empresas lo hayan puesto en funcionamiento en los cursos de teleformación a empleados internos y externos (La Caixa, Intel o Cisco son una pequeña muestra)

Es obvio, pues, que las plataformas virtuales ahorran tiempo a profesores y estudiantes: en la era de Internet ya no hace falta desplazarse hasta la Facultad para ver el tablón de la asignatura y consultar los resultados de las evaluaciones, trabajos o los horarios de tutoría de los profesores. No obstante, Moodle en cualquiera de sus variantes (Ágora o el Sistema de Apoyo a la Docencia, Swad de la Universidad de Granada) abre muchas más posibilidades al permitir subir archivos de audio y vídeo. Uno de los conocimientos disciplinares básicos en los estudios que ocupan este artículo, es tener una buena cultura audiovisual y dominar el significado y las técnicas de este lenguaje, para, después, poder realizar productos audiovisuales (Libro Blanco de los Títulos de Grado en Comunicación, 2005: 229). Ello se consigue analizan- do documentos audiovisuales de muy diversa índole (desde fotos, spots publicitarios, programas de radio y televisión, vídeo clips, hasta cabeceras de series originales susceptibles de narrar una historia en segundos) y dentro de las horas lectivas de clase no da tiempo a contemplarlo todo; sin embargo, si los docentes habilitan en su plataforma telemática una carpeta donde colgar estos recursos, se pueden llevar a cabo prácticas individuales o en grupo que redunden en un amplio acervo de cultura audiovisual del alumnado.

Del mismo modo, es factible habilitar en las zonas comunes, carpetas en las que los mismos estudiantes suban sus archivos con sus propios trabajos: podcasts, fotos, vídeos, etc. Cada una de estas actividades satisface las prácticas de las materias del Grado en Comunicación Audiovisual, favoreciendo el trabajo autónomo y la creatividad del alumnado.

El uso de estas plataformas interactivas tiene un aspecto fundamental: que los alumnos y alumnas aprenden a utilizar un tipo de comunicación específica, mediada por la gestión de contenidos en un entorno digital y multimedia, lo cual se recoge en el Libro Blanco de los Títulos de Grado en Comunicación dentro de los "conocimientos disciplinares (saber): Conocimiento teóricopráctico y aplicación de las tecnologías aplicadas a los medios de comunicación audiovisuales (fotografía, radio, sonido, televisión, vídeo, cine, y soportes multimedia)" (2005: 231). 
Actualmente un gran número de docentes apuesta por el trabajo colaborativo de los alumnos y alumnas por medio de las wikis. Un ejemplo de su repercusión se observa en una de los foros del IV Congreso de la Cibersociedad 2009, donde diversos docentes relatan sus experiencias con este método de aprendizaje constructivista ${ }^{1}$. Las wikis son páginas webs cuyos contenidos se desarrollan de forma colaborativa por las aportaciones de los propios usuarios. La más famosa de las wikis es Wikipedia, muy consultada, por cierto, por los estudiantes de casi todos los niveles académicos, podemos afirmar, por tanto, que el estilo de esta herramienta en línea es fácilmente conocido por cualquier usuario de la web; ahora bien, todas sus posibilidades han sido aprovechadas por el profesor de la Universidad Oberta de Catalunya (UOC), David Gómez Fontanills. Esta experiencia se ha orientado para la asignatura de producción y tratamiento de gráficos por ordenador del Graduado Multimedia de la UOC, aunque es una wiki accesible para cualquiera que esté interesado en el tema, ya sean profesores o alumnos, cualquier persona se puede dar de alta y participar en los foros, ver el trabajo de los estudiantes, acceder al material bibliográfico, enlaces webs, etc. Está hecha con la licencia libre MediaWiki (licencia Copyleft GPL). La mayor ventaja de su uso es que permite un trabajo totalmente colaborativo, al publicar cada estudiante su práctica, el resto de los compañeros complementan y enriquecen cada acti- vidad con aportaciones propias. Las prácticas, al ser abiertas también ayudan al alumnado de otros cursos y niveles. La asignatura está estructurada en varias partes: teoría ("unidades de estudio"), actividades para el aprendizaje y la evaluación (de los temas percepción visual, lenguaje y comunicación visual, diseño gráfico e imagen digital).

Indiscutiblemente, las universidades de educación a distancia están más avanzadas en las herramientas TIC para la teleformación, las cuales son también eficaces en la metodología del EEES.

Existen en la red algunos recursos de $e$ learning específicos para el área de la Comunicación Audiovisual que integran términos y nociones concretas de esta especialidad con recursos interactivos para el aprendizaje autónomo, actividades para favorecer la capacidad de obtención de la información o relacionar conceptos de forma crítica y significativa. En este caso reseñamos el portal Alfamedia, dirigido por la Catedrática de de Documentación y Profesora de la Facultad de Comunicación y Documentación de la Universidad de Granada, María Pinto. Para los alumnos y alumnas del primer curso de Comunicación Audiovisual puede resultar muy instructivo dado que, por un lado, las nociones de esta área concreto sirven de complemento básico e introductorio fundamental para quienes tomen contacto por primera vez con los media, industrias culturales y sus productos etc., mientras que por otro lado, se dan las claves para que los recién llega- 
dos a la enseñanza superior vayan familiarizándose con los nuevos métodos de estudio.

El portal Alfamedia tiene como objetivo:

Capacitar al estudiante en las competencias del aprendizaje visual a través de la imagen - tan importante en el contexto de las nuevas tecnologías - mediante el uso de recursos hipermedia, favoreciendo el manejo de cualquier tipo de recurso informacional, con el fin de favorecer un aprendizaje integrado e innovador, autónomo y reflexivo (Pinto, 2009).

Dentro de los contenidos de este portal destaca información tan importante como habilidades y métodos que los estudiantes deben manejar con soltura relativas a la búsqueda de información procedente de diversas fuentes o hiperenlaces para ampliar en temas transversales y complementarios a la educación superior (competencias en la comunicación escrita y oral, técnicas de estudio, etc.)

Otro portal que merece la pena destacar en este epígrafe es el "Portal de la UAB", donde se "ofrece información y documentación especializada en los diferentes ámbi- tos de la comunicación, orientada a los investigadores, estudiantes y profesionales de la Comunicación, prioritariamente de América Latina, España y Cataluña”. Es un sitio web muy completo con secciones dedicadas a investigaciones, entrevistas, conferencias, congresos y simposios, y una amplia variedad de recursos en línea acerca de cine, radio, televisión, periodismo, etc., en español y en inglés. Entre sus objetivos principales destacan la selección y sistematización de la información de la red sobre comunicación y el fomento de la investigación en ciencias de la comunicación.

Los recursos aquí mostrados son un pequeño ejemplo de lo que ya hay, y de cómo se pueden poner en práctica para conseguir los objetivos del EEES en lo que respecta a la forma de "regular el proceso de aprendizaje” (Cabero Almenara, 2007: 27), para facilitar los cambios que la convergencia europea propone en los contenidos del Grado de Comunicación Audiovisual. En sí mismas, estas herramientas no son ni buenas ni malas, por lo que es necesario tener en cuenta ciertas consideraciones críticas con respecto a esta metodología.

\section{Un punto de vista crítico: mitos sobre las NNTT}

La primera crítica que se suele hacer a las NNTT y TIC es que éstas, por sí solas, no generan conocimiento. Es "el mito de la información versus el conocimiento": "El mero hecho de usar un multimedia o Internet no implica un proceso de innovación, si previamente no se ha reflexionado sobre los propios medios y sobre qué innovación 
se pretende lograr” (Aparici, 1990). Efectivamente, los docentes que emplean estos métodos deben hacer una planificación previa acerca de los objetivos, habilidades procedimentales y actitudinales que se quieren desarrollar en el alumnado. Por lo que, la información, para que se trasforme en conocimiento, debe ser procesada y convertirse en objeto de crítica, discusión y reflexión, pues si la información "no se procesa, no se convierte en conocimiento" (Aparici, 1990). En este sentido, el profesorado pasa a ser un tutor o guía del aprendizaje, no un mero emisor de información, adoptando el papel que el EEES otorga a los docentes. Otro error en el que no se debe incurrir es en "el mito del aprendizaje mágico", es decir, las NNTT sin más no producen el aprendizaje en el alumno, sino que se han de integrar en un planteamiento docente coherente. Tampoco implica que usar Internet o recursos multimedia vaya a suplir las carencias de conocimiento de los docentes, es más, en la mayoría de los casos lo evidencia. De nuevo se impone la planificación y el sentido común: no utilizar los recursos por usarlos, hay que hacerlo sabiendo el porqué y para qué de su uso. Otra cuestión muy importante es el dar por hecho que los alumnos y alumnas conocen a la perfección el entorno digital que se utiliza y los objetivos con los que se utiliza. Como señala Ilona Kovács (Rodríguez Castro, 2004) tan solo una pequeña minoría de los usuarios "utiliza los nuevos medios rentablemente, los que están ya en situa- ción ventajosa; la mayoría, utiliza su acceso ocasional a las TIC para diversión, sin redundar en una verdadera formación profesional o cultural” (Kovács, 1998). Por lo tanto es necesario trasmitir a los y las estudiantes el enorme potencial de las NNTT y TIC para que sean capaces de sacarle todo el partido posible en todas sus facetas: "De esto tenemos sobrados ejemplos entre nuestros alumnos, manejadores habilísimos de ordenadores y videojuegos, sin mínima base tan siquiera para entender la realidad que les circunda ni acceder, con alguna preparación, al mundo laboral” (Rodríguez Castro, 2004). Aquí tiene especial relevancia la madurez del alumnado, pues este planteamiento de aprendizaje autónomo requiere una lucidez intelectual que, quizás, con 18 años de edad aún no esté completamente desarrollada. Por esta razón, en el apartado anterior se incluyó un portal, Alfamedia, que contiene actividades interactivas para fomentar las típicas habilidades del aprendizaje autónomo, el gran tema transversal a todos los planes de estudio en el EEES. Por ello, un aprendizaje personalizado con el profesor como tutor es elemental, pero para lograrlo, se necesita contar con grupos de trabajo reducidos a fin de que la evaluación o seguimiento continuo sea posible. De la misma forma, el trabajo en grupo y cooperativo, a base de foros de discusión y debate es parte del apoyo que un alumno o alumna con menor capacidad crítica puede aprovechar y aprender de otros compañeros más aventa- 
jados (recordemos que Moodle tiene chats, wikis y foros de discusión que se pueden crear y moderar ad hoc).

Finalmente, si se planteó más arriba que estos recursos interactivos en línea podían servir para que la comunicación entre profesores-alumnos y entre los mismos docentes fuera más fluida, hay que intentar no caer en la "atomización tecnológica": muchas plataformas puede conducir a que la información se pierda al no estar centralizada. Es aconsejable acordar el uso de una en concreto, por todos o la mayoría de los profesores o, en su defecto, considerar la posibilidad de abrir cursos a invitados para tener la información accesible.

\section{Conclusiones}

A lo largo del artículo se han intentado desglosar las claves de innovación docente que la convergencia europea introduce en los planes de estudio de la enseñanza universitaria.

El más importante es el intento de adecuar la metodología didáctica a la sociedad actual y construir en el alumnado la filosofía de un aprendizaje a lo largo de su vida que pueda servirles tanto en su etapa universitaria como laboral. Aprender es una experiencia muy gratificante y enseñar conocimientos útiles, despertando la curiosidad y la capacidad crítica en las y los estudiantes debe ser la meta diaria de un docente.

No obstante, es necesario resaltar que, la clave del aprendizaje continuo debe, hoy más que nunca, ser también adoptado por los propios docentes para sacar el máximo partido posible a las NNTT y TIC, adaptadas a los requisitos de los nuevos grados. Estas herramientas, como hemos visto en los ejemplos concretos, son realmente versátiles y útiles en los estudios de Comunicación Audiovisual.

Aun así, el principal objetivo de este artículo no ha sido trasmitir una idea de fácil "tecnofilia", donde los ordenadores se vislumbran como panacea para paliar las dificultades del proceso enseñanza y aprendizaje, sino que se ha tratado de hacer un compendio crítico y razonado de los pros y contras que supone incorporar las herramientas tecnológicas en el aula, siempre dentro del marco que el EEES traza.

Tampoco se ha de perder de vista que los métodos tradicionales siguen teniendo vigencia: las bibliotecas y los libros impresos en papel son fundamentales, pero la forma de consultar las bases de datos bibliográficas, por ejemplo, son ahora más cómodas y fáciles gracias a las NNTT.

Por último, queremos destacar que los profesores y profesoras han de mantener una perspectiva abierta hacia las TIC y NNTT, pero sobre todo, han de saber que utilizarlas conlleva un considerable esfuer- 
zo de planificación, y que utilizar el ordenador para hacer presentaciones multimedia en las clases magistrales no es la innova- ción docente ni tecnológica que el EEES plantea.

\section{Referencias}

Alfamedia. Pinto, M. (Dir.) [En línea] Recuperado el día 8 de diciembre de 2009, de http://www.mariapinto.es/alfamedia/

Agencia Nacional de la Calidad y Acreditación. Libro Blanco de los Títulos de Grado en Comunicación (2005). [En línea]. Recuperado el 29 de noviembre, de http://www.aneca.es/publicaciones/librosblancos.aspx

Aparici, R. (1999). Mitos de las nuevas tecnologías y de la educación a distancia. [En línea] Recuperado el día 8 de diciembre, de

http://dialnet.unirioja.es/servlet/fichero articulo? codigo $=127589 \&$ orden $=0$

Boletín Oficial del Estado (2003, 18 de septiembre), Real Decreto 1125/2003. [En línea]. Recuperado el día 6 de diciembre, de

http://www.boe.es/boe/dias/2003/09/18/pdfs/ A34355-34356.pdf

Cabero Almenara, J. (Dir.) (2007). Formación del profesorado universitario en estrategias metodológicas para la incorporación del aprendizaje en red en el espacio de educación superior (EEES). [En línea] España:

Universidad de Sevilla. Acceso proporcionado por la Universidad de Granada, Recuperado el día 1 de diciembre, de

http://site.ebrary.com/lib/univgranada/docDetail. action?docID $=10169378 \&$

Castells, M. (2007). La transición a la sociedad Red.

Barcelona: Ariel

Declaración de Bolonia. [En línea]. Recuperado el día 2 de diciembre, de

http://www.crue.org/export/sites/Crue/procbol onia/documentos/antecedentes/2. Declaracixn de Bolonia.pdf

Declaración de Praga. [En línea]. Recuperado el día 2 de diciembre, de http://www.ual.es/personal/nperdu/praga.html
Foros del IV Congreso de la Cibersociedad 2009: crisis analógica futuro digital. Asunto: GRF Wiki UOC: trabajo colectivo, colaboración y evaluación [En línea] Recuperado el día 10 de diciembre de 2009, de:

http://www.cibersociedad.net/congres2009/es/fo rums/posts/grf-wiki-uoc-trabajo-colectivocolaboracion-y-evaluacion/30/

Gimeno Sacristán, J. (Coor.). (2008). Educar por competencias ¿qué hay de nuevo? Madrid: Morata

Gómez Fontanills, D. (2009). GRF Wiki UOC: trabajo colectivo, colaboración y evaluación. Ponencia presentada en el IV Congreso de la Cibersociedad 2009: crisis analógica futuro digital. [En línea] Recuperado el día 10 de diciembre de 2009, de:

http://www.cibersociedad.net/congres2009/es/co ms/grf-wiki-uoc-trabajo-colectivo-colaboracion-yevaluacion/909/

González Boticario, J. y Gaudioso Vázquez, E. (2003). Sistemas interactivos de enseñanza / aprendizaje. Madrid: Sanz y Torres

González, J y Wagenaar, R. (Ed.). (2003). Tuning Educational Structures in Europe Informe Final Fase Uno.[En línea] Recuperado el día 5 de diciembre, de

http://www.relint.deusto.es/TUNINGProject/spa nish/doc fase $1 /$ Tuning $\% 20$ Educational.pdf

Molist, M. (2008, 4 de diciembre). Moodle llena la geografía española de campos virtuales. El País. [En línea] Recuperado el día 6 de diciembre, de

http://www.elpais.com/articulo/portada/Moodle /llena/geografia/educativa/espanola/campus/virtu ales/elpepisupcib/20081204elpcibpor 1/Tes/ 
Pestano Rodríguez, J. M. (1999). Aportaciones de las nuevas tecnologías en el ámbito de la comunicación audiovisual en las instituciones. Revista Latina de Comunicación Social, 23. Recuperado el 4 de diciembre de 2009 de:

http: / / www.ull.es/publicaciones/latina/a1999bno /14pestano.html

Portal de la Comunicación. El portal de los estudios de comunicación. [En línea] Consulta del día 6 de diciembre de 2009, de http://www.portalcomunicacion.com/esp/home.a sp
Rodríguez Castro, F. (2004). Las reformas educativas y el mito de las nuevas tecnologías. En Colectivo Baltasar Gracián. Revista Crisis [En línea] Número 6 (2004, julio). Recuperado el día 6 de diciembre, de

http: / / www.colectivobgracian.com/revista/revista $\mathrm{htm} /$ numero\%20 $\%$ seis/las reformas educativas y e $\underline{\text { l mit.htm }}$

Rodríguez Escancio, I. (Ed.). (2008). El nuevo perfil del profesor universitario en el EEES: claves para la renovación metodológica. Valladolid: UEMEC

\section{NOTAS}

${ }^{1}$ El constructivismo es un estilo pedagógico en el que "el conocimiento debe ser construido por el aprendiz, pieza por pieza” (González Boticario y Gaudioso Vázquez, 2003: 7) Moodle se basa también en la pedagogía constructivista. 